\title{
Biopolymer Nanocomposite Films Reinforced with Nanocellulose Whiskers
}

\author{
Amit Saxena ${ }^{1}$, Marcus Foston ${ }^{1}$, Mohamad Kassaee ${ }^{2}$, Thomas J. Elder ${ }^{3}$, and Arthur J. Ragauskas ${ }^{1, *}$ \\ ${ }^{1}$ School of Chemistry and Biochemistry, Georgia Institute of Technology, Atlanta, GA, 30332 \\ ${ }^{2}$ School of Chemical and Biomolecular Engineering, Georgia Institute of Technology, Atlanta, GA \\ ${ }^{3}$ USDA-Forest Service, Southern Research Station, Pineville, LA, 71360
}

\begin{abstract}
A xylan nanocomposite film with improved strength and barrier properties was prepared by a solution casting using nanocellulose whiskers as a reinforcing agent. The ${ }^{13} \mathrm{C}$ cross-polarization magic angle spinning (CP/MAS) nuclear magnetic resonance (NMR) analysis of the spectral data obtained for the NCW/xylan nanocomposite films indicated the signal intensity originating from xylan-cellulose interactions. Qualitatively, the spectral data obtained for the NCW/xylan nanocomposite films indicated that the amount of xylan adsorbed to cellulose increases with the addition of NCW. In an attempt to quantify this effect, non-linear least-squared spectral line fitting was used to deconvolute the adsorbed xylan peak at $\sim 82 \mathrm{ppm}$. The peak intensity ratio of adsorbed xylan peak and xylan $\mathrm{C}_{1}$ peak, which represents the total amount of xylan increases suggesting that upon the addition of NCW, the amount of adsorbed xylan increases. In an effort to further infer the structure-property relationships associated with the observed strength and barrier properties, ${ }^{1} \mathrm{H} N M R \mathrm{~T}_{2}$ relaxation experiments were also conducted to investigate the change in the nature of carbohydrate-water interactions as a result of NCW incorporation. Water adsorbed into the $50 \%$ nanocomposite film had significantly shorter relaxation times with respect to the control xylan/sorbitol and all other $\mathrm{NCW} / x y l a n$ nanocomposite films. Additionally, X-ray diffraction of the nanocomposite films showed increased levels of crystalline material in the nanocomposites due to NCW addition.
\end{abstract}

Keywords: Nanocomposites, Nanocellulose Whiskers, Mechanical Properties, Barrier Properties.

\section{INTRODUCTION}

Environmental concerns arising from the use of nondegradable petroleum-based plastics have resulted in the search for suitable substitutes. ${ }^{1-4}$ In recent years, increasing societal concerns have accelerated efforts to develop new ways to utilize biopolymers such as polysaccharides, proteins and lipids to create biodegradable films due to their ready availability, sustainability and reduced end-oflife environmental concerns. ${ }^{5-8}$ Many naturally occurring polysaccharides and their derivatives such as starch, cellulose, chitosan, alginates, carrageenan, pectinate, and various naturally occurring gums have been proposed for use in coating and film formulations.

Typically, polysaccharide films possess poor mechanical and barrier properties, ${ }^{9-10}$ and plasticizers are normally added to improve the strength of the films and aid in stabilizing the film network. ${ }^{11-12}$ Xylan, which is one the most common hemicelluloses and the most abundant

\footnotetext{
*Author to whom correspondence should be addressed.
}

polysaccharide in nature after cellulose, is an attractive resource for film production. ${ }^{13-15}$ Recently, acetylated galactoglucomannan (AcGGM) hemicellulose was found to be an alternative material for making new renewable barrier materials. ${ }^{16}$ The oxygen barrier permeability of the AcGGM films was found to be similar to, or lower than, the values reported for oxygen barrier films made from glucuronoxylan ${ }^{17}$ and other polysaccharides, such as starch, ${ }^{18}$ chitosan ${ }^{19}$ and mixtures of various polysaccharides. ${ }^{20}$ Glucuronoxylan, isolated from aspen wood, when plasticized with xylitol or sorbitol has been used to produce films that exhibit improved strength and oxygen barrier properties with respect to control glucuronoxylan films. ${ }^{21-22}$ Hartman et al. reported oxygen permeability values of $2.0 \mathrm{~cm}^{3} \mu \mathrm{m} \mathrm{m}^{-2}$ day $^{-1} \mathrm{kPa}^{-1}$ for GGM-sorbitol film. ${ }^{16}$ Miranda et al. studied water vapor transmission values of chitosan film plasticized with sorbitol, glycerol or polyethylene glycol reporting values from $1.3-1.5 * 10^{-3}\left[\mathrm{~g} /\left(\mathrm{m}^{2} \cdot \mathrm{h} \cdot \mathrm{Pa}\right)\right] .^{23}$ The goal was to decrease the water vapor permeability and to improve the mechanical properties of chitosan films. Miranda et al. 
also discussed that in hydrophilic films, water molecules interact with polar groups in the film structure, causing plasticization or swelling, which, in turn results in varying permeability. $^{23}$

Films made from these polysaccharides are brittle and therefore to form cohesive films requires plasticizers such as sorbitol and xylitol. However, even with the plasticizers, the mechanical properties of these films have been considered to be poor. Nanocellulose whiskers (NCW) have been studied as reinforcements of various synthetic and natural polymer matrices ${ }^{24-26}$ to improve the strength properties of the nanocomposites. They have a high bending strength of $10 \mathrm{GPa}$, an elastic modulus of $143 \mathrm{GPa}^{27,28}$ and forms a rigid hydrogen-bonded network. ${ }^{6}$ The properties of nanocomposite materials and the enhancement of these properties, depend not only on their individual components but also on the interaction between the matrix and the reinforcing phases and their morphology. ${ }^{29,30}$ Recent studies by Saxena et al. have shown that xylan films reinforced with NCWs can reduce water and oxygen permeability by greater than $+70 \%$ while increasing physical strength properties by $+50 \%$ with respect to control xylan/sorbitol film by the addition of $5-10 \%$ of NCWs. ${ }^{31-33}$

The main objective of this paper is to utilize X-ray diffraction (XRD) and nuclear magnetic resonance (NMR) techniques to study the structure-property relationships of xylan films reinforced with NCWs. A critical issue to probe is the interactions of NCWs with the film matrix because of the significantly increased matrix-filler interfacial area typically associated with nanocomposite. In addition, we used NMR $T_{2}$ relaxation experiments to investigate the change in the nature of carbohydratewater interactions as a result of $\mathrm{NCW}$ incorporation. These results will facilitate an improved understanding of the mechanisms involved in the superior barrier and mechanical properties of xylan-NCW nanocomposite films previously reported. ${ }^{31-33}$

\section{EXPERIMENTAL DETAILS}

\subsection{Materials}

Oat spelt xylan and sorbitol were obtained from Aldrich and used as received. Oat spelt xylan was determined to contain $81.0 \%$ xylose, $9.8 \%$ arabinose, $7.6 \%$ glucose, $1.4 \%$ galactose and $0.2 \%$ of mannose. The molecular weight of the xylan employed was $M_{\mathrm{n}}$ of $2.45 \times 10^{3}$ and $M_{\mathrm{w}}$ of $1.42 \times 10^{5} \mathrm{~g} / \mathrm{mol}$ as determined by gel permeation chromatography and referenced to six narrow standard polystyrene calibration curve. A dry commercial elemental chlorine-free (ECF) bleached softwood (SW) kraft pulp manufactured in the southeastern US was used as received. Dialysis tubes were purchased from Spectrum Labs, MWCO $50 \mathrm{~K}$. All other reagents and solvents were purchased from Aldrich and used as received.

\subsection{Preparation of Nanocellulose Whiskers}

NCWs were prepared using the procedure published by Saxena et al. ${ }^{33}$ In brief, softwood kraft pulp was ground in a Wiley mill to pass through a 20-mesh screen. Acid hydrolysis of the milled pulp was accomplished with $64 \mathrm{wt} \%$ sulfuric acid at $45^{\circ} \mathrm{C}$ for $45 \mathrm{~min}$ with stirring. The reaction was halted by adding a 10 -fold excess of deionized (DI) water. After standing overnight, the water was decanted and the settled cellulosics were collected with a minimal amount of water. Excess acid was removed with a water wash $(200 \mathrm{ml})$, centrifuged initially at $3000 \mathrm{rpm}$ for $20 \mathrm{~min}$ and then at $11,000 \mathrm{rpm}$ for $20 \mathrm{~min}$. The aqueous phase was discarded and the wash cycle was repeated two additional times. The solid sample was then dialyzed with a Spectra/Por membrane (MWCO $50 \mathrm{~K}$ ) against water for several days until the whisker slurry achieved a neutral $\mathrm{pH}$. Sonication was performed on the neutral nanocrystalline cellulose solution for $35 \mathrm{~min}$ with an Ultrasonic Processor GEX-500 sonicator with a titanium alloy probe (tip diameter $1.27 \mathrm{~cm}$ ) at 28 watts power input while immersed in an ice bath. The average yield of nanocrystalline cellulosic was $35 \%$. The bulk charge for the starting cellulose fibers was found to be $3.82 \mathrm{mmol}$ carboxylate groups/100 $\mathrm{g}$ and the bulk charge for the NCWs was found to be $9.73 \mathrm{mmol}$ acid groups/100 g. ${ }^{33}$ By AFM analysis, the sulfonated NCWs were observed to have rod like structure with an average length of 150-200 $\mathrm{nm}$ and a width of less than $20 \mathrm{~nm}$.

\subsection{Preparation of Nanocomposite Films}

The detailed procedure for preparing the xylan, sorbitol and NCW nanocomposite was discussed in previously published papers. ${ }^{32,33}$ In brief, xylan nanocomposite films were formed by adding an aqueous suspension $(35.00 \mathrm{ml})$ of sulfonated whisker suspension to xylan $(0.25 \mathrm{~g})$. The solid whisker content in the whisker suspension used was $0.0,5.0,10.0,25.0,50.0 \mathrm{wt} \%$ of the total mixture of sorbitol, xylan, and NCWs. Sorbitol $(0.25 \mathrm{~g}, 1.37 \mathrm{mmol})$ was added to the mixture with stirring and the mixture was heated to $95{ }^{\circ} \mathrm{C}$ for $15 \mathrm{~min}$. The solution was then poured into polystyrene petri dishes and allowed to dry at room temperature for three days.

\subsection{Characterization}

\subsection{Nuclear Magnetic Resonance (NMR) Analysis}

NMR samples were prepared with films conditioned for $48 \mathrm{~h}$ at $100 \%$ relative humidity and then packed into 4-mm cylindrical ceramic MAS rotors. Solid-state NMR measurements were carried out on a Bruker Avance- $400 \mathrm{MHz}$ spectrometer operating at frequencies of $100.55 \mathrm{MHz}$ for ${ }^{13} \mathrm{C}$ NMR experiments using a 4-mm Bruker doubleresonance MAS probehead at spinning speeds of $10 \mathrm{kHz}$. 
CP/MAS experiments utilized a $5 \mu \mathrm{s}\left(90^{\circ}\right)$ proton pulse, $2.0 \mathrm{~ms}$ contact pulse, $4 \mathrm{~s}$ recycle delay and $8 \mathrm{~K}$ scans. A $10 \mathrm{~Hz}$ exponential line-broadening apodization was applied. Line-fitting analyses of the spectra were performed using NUTS NMR Data Processing software (Acorn NMR, Inc).

\subsubsection{XRD (X-ray Diffraction)}

Wide Angle X-ray Diffraction (WAXD) patterns were obtained on a Philips X'pert diffractometer equipped with $\mathrm{X}$ 'celerator using $\mathrm{Cu} \mathrm{K} \alpha$ radiation. The patterns were recorded on the prepared films at $45 \mathrm{kV}$ and $40 \mathrm{~mA}$. The pattern was taken over a range of $2 \theta$ from 5 to $40^{\circ}$ with a step size of $0.02^{\circ}$. The films were prepared as discussed previously and were put on low background holder for XRD measurement.

\section{RESULTS AND DISCUSSION}

This study utilizes X-ray diffraction (XRD) and nuclear magnetic resonance (NMR) techniques to study the structure-property relationships of xylan films reinforced with NCWs which will facilitate an improved understanding of the mechanisms involved in the superior barrier and mechanical properties of rigid hydrogen bonded xylanwhisker nanocomposite films previously reported..$^{31-33} \mathrm{~A}$ control xylan/sorbitol film was prepared from a 1:1 weight ratio of xylan and sorbitol. The morphology of the nanocomposite films and the size distribution of nanocellulose whiskers were fully characterized using SEM and AFM and previously reported by Saxena et al. ${ }^{31-33}$ Nanocellulose whiskers were shown to have a rod like shape with length values of $150-250 \mathrm{~nm}$ and width on the order of $5-15 \mathrm{~nm}^{33}$

\subsection{Solid-State ${ }^{13} \mathrm{C}$ CP/MAS NMR Analysis on Xylan Nanocomposite Films}

To characterize the interaction of NCWs with the matrix, NMR analysis on the nanocomposite films were performed. Solid-state ${ }^{13} \mathrm{C}$ CP/MAS NMR analysis of a control xylan/sorbitol film, NCW and NCW/xylan nanocomposite films is shown in Figure $1 .{ }^{13} \mathrm{C}$ NMR chemical shifts of the starting xylan and NCWs agreed with literature values ${ }^{34-37}$ and could be readily assigned in the spectra of the nanocomposite films. The ${ }^{13} \mathrm{C} \mathrm{CP/MAS}$ NMR spectra of the NCWs showed the typical spectra of crystalline cellulose and based on the integration of the cellulose $\mathrm{C}_{4}$ peak region, the NCWs had a crystallinity of $\sim 64 \%$.

Newman et al. has made specific assignments for the cellulose $\mathrm{C}_{4}$ spectral resonances arising from cellulose crystallite interiors, crystallite surfaces and amorphous regions. ${ }^{38}$

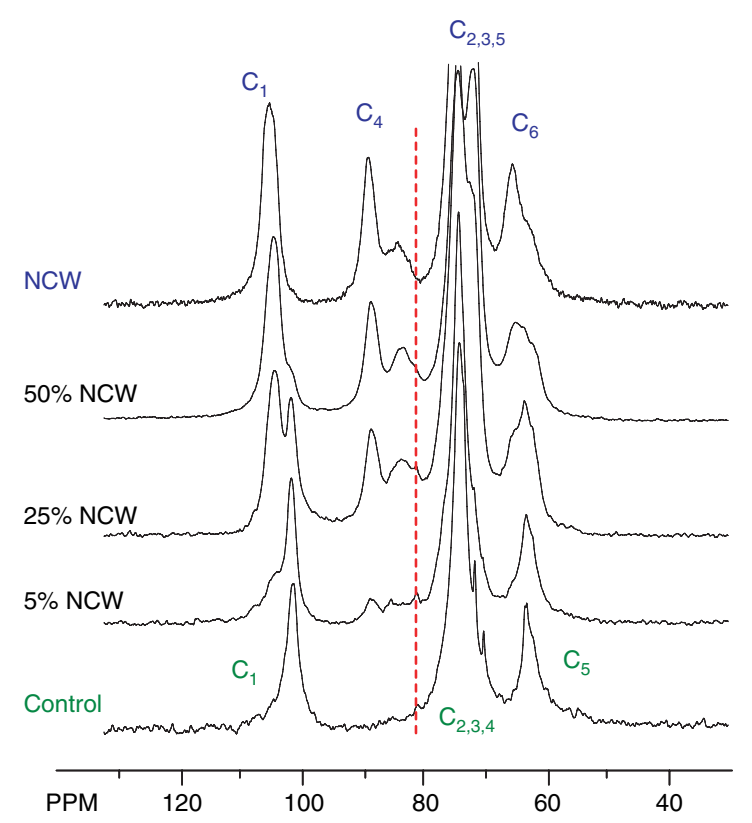

Fig. 1. ${ }^{13} \mathrm{C}$ spectra of $\mathrm{NCW} / \mathrm{xylan}$ nanocomposite films of increasing NCW content conditioned under $100 \%$ RH for $48 \mathrm{~h}$.

Recent results reported by Lopez et al. suggested that the enthalpic interaction exists in xyloglucan-cellulose whiskers system. ${ }^{39}$ The results also suggested that the strong xyloglucan-cellulose interactions are governed by several types of interactions, including hydrogen bonding and hydrophobic interactions. ${ }^{39}$

As shown in both Figures 1 and 2 , the $C_{1}$ peak of cellulose and xylan is $\sim 105$ and 102 ppm, respectively. The analysis of the both the $\mathrm{C}_{4}$ and $\mathrm{C}_{1}$ peak regions of xylan/cellulose are unobstructed by resonances from sorbitol which only appear 74-63 ppm. Figure 2 shows the non-linear least-squared line-fitting of the $\mathrm{C}_{1}$ region for the ${ }^{13} \mathrm{C}$ spectra of $25 \% \mathrm{NCW} / \mathrm{xylan}$ nanocomposite film. A primarily Gaussian line-shape was applied to the carbon signal attributed to the $\mathrm{C}_{1}$ peak of cellulose with a constant line-width of $294 \mathrm{~Hz}$ (based on the neat NCW spectrum), while a Lorentzian weighted line seemed to describe the signal from the $\mathrm{C}_{1}$ peak of xylan. Though the spectrum in Figure 2 suggests these peaks are well-resolved, the other nanocomposite films required deconvolution by line-fitting due to the highly reduced contribution of either NCWs or xylan in the 5 and $50 \%$ NCW nanocomposite films. Larsson et al. reviewed the interaction of cellulose and hemicellulose using CP/MAS ${ }^{13} \mathrm{C}-\mathrm{NMR}$ spectroscopy ${ }^{34}$ and our results are consistent with this report with respect to the types of line-shape and peak chemical shifts.

Larsson et al. reported that for 70/30\% (w/w) mixture of cellulose and xylan, when heated in the presence of water an additional resonance not present in either the spectra of cellulose or xylan appeared. ${ }^{34}$ It appears that cellulose-xylan interactions increases as water is removed from the system in the heating process which may cause 


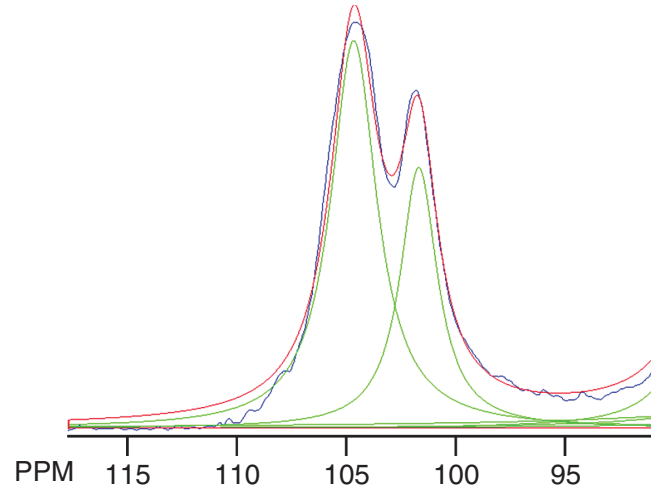

Fig. 2. The non-linear, least-squared, spectral fitting of the $\mathrm{C}_{1}$ region for the ${ }^{13} \mathrm{C}$ spectra of $25 \% \mathrm{NCW} / \mathrm{xylan}$ nanocomposite film conditioned under $100 \% \mathrm{RH}$ for $48 \mathrm{~h}$. Green lines $=C_{1}$ peak of cellulose and xylan; Red lines $=C_{1}$ peak summed fit of cellulose and xylan and; Blue line $=$ real spectra.

a formation of a strong hydrogen bonding between xylan and cellulose. ${ }^{34}$ This signal was observed at $\sim 82 \mathrm{ppm}$, slightly overlapping the signal from inaccessible cellulose fibril surfaces at $\sim 84 \mathrm{ppm}$. The authors concluded that this additional peak must be attributed to xylan adsorbed to cellulose and would be accompanied by a loss of signal intensity from cellulose at accessible fibril surfaces and an increase in signal intensity from cellulose at inaccessible fibril surfaces due to the 'coverage' by xylan. ${ }^{34}$ Qualitatively, the obtained spectra of the NCW/xylan nanocomposite films seen in Figure 1 demonstrate a similar affect. The relative signal intensity at $\sim 81.7 \mathrm{ppm}$ is appreciably higher in the spectra of all the nanocomposite films with respect to the spectra of the control xylan/sorbitol film or neat nanocellulose whisker. In an attempt to quantify this effect, non-linear least-squared spectral line fitting was used to deconvolute the adsorbed xylan peak at $\sim 82 \mathrm{ppm}$. The results are presented in Table I, as a ratio of $\delta_{82 \mathrm{ppm}}: \delta_{102 \mathrm{ppm}}$ with respect to the xylan $\mathrm{C}_{1}$ peak, which represents the total amount of xylan present in the nanocomposite. The ratio seen in Table I has been adjusted, subtracting intensity from the $82 \mathrm{ppm}$ region based on the deconvoluted intensity of cellulose $C_{1}$ peak and a predetermined peak intensity ratio at $\delta_{82 \mathrm{ppm}}: \delta_{105 \mathrm{ppm}}$ for neat NCW. This was done in an effort to account for the overlapping the signal from inaccessible cellulose fibril surfaces. This ratio suggests that upon the addition of $50 \% \mathrm{NCW}$, the amount of adsorbed xylan increases by a factor of three.

\subsection{NMR $T_{2}$ Relaxation Experiments on Xylan Nanocomposite Films}

NMR $T_{2}$ relaxation experiments can provide information pertaining to the molecular mobility within a system. ${ }^{1} \mathrm{H}$ Carr-Purcell-Meiboom-Gill (CPMG) experiments were used to investigate the change in the nature of carbohydrate-water interactions as a result of NCWs incorporation. $T_{2}$ relaxation values probe how fast a nuclei loses transverse magnetization. In this experiment, the signal intensity decays as a function of local inhomogeneities in the magnetic field mainly due to perturbation by nuclei through space or dipolar interactions, and this signal attenuation or the characteristic relaxation rate for this process is called the spin-spin relaxation (i.e., $T_{2}$ ). Basically, the faster the rate of the decay, the more rigid or fewer degrees of freedom the chemical group associated with that decay has. ${ }^{40}$ Spin-spin (T2) relaxation of water adsorbed in western red cedar and Douglas fir sapwood have been studied in the past ${ }^{41,42}$ and recently Marcus et al. ${ }^{43}$ used spin-spin (T2) relaxation to study the interaction of cellulose and water. We used a similar method to study the ${ }^{1} \mathrm{H} T_{2}$ relaxation of water adsorbed into the NCW nanocomposite system to infer the structure-property relationships with respect to the enhanced strength and barrier properties observed for these xylan-nanocomposite films. The analysis of wide $T_{2}$ distributions using a single model can be difficult. One technique to resolve this issue, while also showing any change in $T_{2}$ relaxation, is to extract the distribution of relaxers or exponential decays from the $T_{2}$ data using an inverse Laplace transform (ILT) routine. $^{22}$ Figure 3 shows the relaxation rate distributions of adsorbed water in the control xylan/sorbitol film, NCWs and nanocomposite films. The $T_{2}$ distribution for the control xylan/sorbitol film indicates at least two distributions of water exist. There are peaks centered at $\sim 12$ and $130 \mathrm{~ms}$. The shorter relaxation time distribution suggests this water is on average less mobile, having less degrees of freedom. This can be interpreted as meaning the water associated with this distribution is either within a much more confined volume or pore space and/or there exist increased interactions with the xylan substrate which adequately retards the molecular dynamics of the water with respect to the water associated with the peaks found at $130 \mathrm{~ms}^{43}$

Between 10 and $25 \%$ whisker content the major distribution of peaks originally centered at $10 \mathrm{~ms}$ shifts to larger $T_{2}$ times indicating the strength of the carbohydrate-water interactions in the xylan matrix are becoming weaker. ${ }^{43}$ However, the water adsorbed into the $50 \% \mathrm{NCW}$ film has significantly shorter relaxation time with respect to the control xylan/sorbitol, 5, 10 and 25\% CNW/xylan nanocomposite films with a very narrow distribution. This shift strongly correlates with the change in the amount of adsorbed xylan discussed previously. This would suggest that either the carbohydrate-water interactions in this system are dominated by that of CNWs, a radical reduction in the pore size distribution ${ }^{33}$ and/or there was a significant change in strength of the carbohydrate-water interactions in the xylan matrix for the $50 \% \mathrm{NCW}$ film with respect to all the other films.

X-ray diffraction (XRD) studies on the nanocomposite system can also be used to infer the structure-property relationships with respect to the observed physical properties 
Table I. Non-linear least-squared spectral fitting results of the $\mathrm{C}_{1}$ of cellulose and xylan region for the ${ }^{13} \mathrm{C}$ spectra of the NCW/xylan nanocomposite films conditioned under $100 \% \mathrm{RH}$ for $48 \mathrm{~h}$.

\begin{tabular}{|c|c|c|c|c|c|c|c|}
\hline \multirow[b]{2}{*}{ Sample } & \multicolumn{3}{|c|}{ Peak at $104.9 \mathrm{ppm}$} & \multicolumn{3}{|c|}{ Peak at $101.9 \mathrm{ppm}$} & \multirow[b]{2}{*}{ Adjusted $\delta_{82 \mathrm{ppm}}: \delta_{102 \mathrm{ppm}}$} \\
\hline & Relative intensity & FWHM $(\mathrm{Hz})$ & $\%$ Lorentzian & Relative intensity & FWHM (Hz) & $\%$ Lorentzian & \\
\hline Control film & & & & & 231 & 100 & 0.17 \\
\hline NCW & & 295 & 36 & & & & \\
\hline $5 \% \mathrm{NCW}$ & 32 & 295 & 36 & 68 & 186 & 57 & 0.17 \\
\hline $25 \% \mathrm{NCW}$ & 56 & 295 & 36 & 54 & 208 & 90 & 0.34 \\
\hline $50 \% \mathrm{NCW}$ & 92 & 295 & 36 & 8 & 126 & 67 & 0.49 \\
\hline
\end{tabular}

previously observed for these nanocomposite films. ${ }^{44,21}$ Lagaron et al. discussed the role of crystalline structure of polymers and emphasized that high crystallinity in semi-crystalline polymer systems improves barrier properties for polymers used in packaging application. ${ }^{45}$ The present paper uses XRD technique to study xylan films reinforced with NCWs, initially comparing XRD results of dry mechanically mixed film components (xylan, sorbitol and NCWs) with a nanocomposite film formed by dissolution and solution casting (as described in the experimental section) which facilitates intimate blending and the formation of a strong hydrogen bonded structures. ${ }^{2,22}$

In Figure 4, the effect of film formation on the overall film crystallinity is shown. Xylan particles show distinctive semi-crystalline characteristics with a broad peak around $19.3^{\circ}$. The sorbitol particles, used as stabilizer in making the xylan film, are very crystalline (see Fig. 4). The diffractograms of sorbitol show sharp individual peaks, whereas the pure xylan polymer has broader peaks (see Fig. 4) which is in agreement with literature ${ }^{21}$ which shows pure low molecular weight plasticizers give rise to sharp distinct peaks, whereas the xylan has broader peaks resulting from

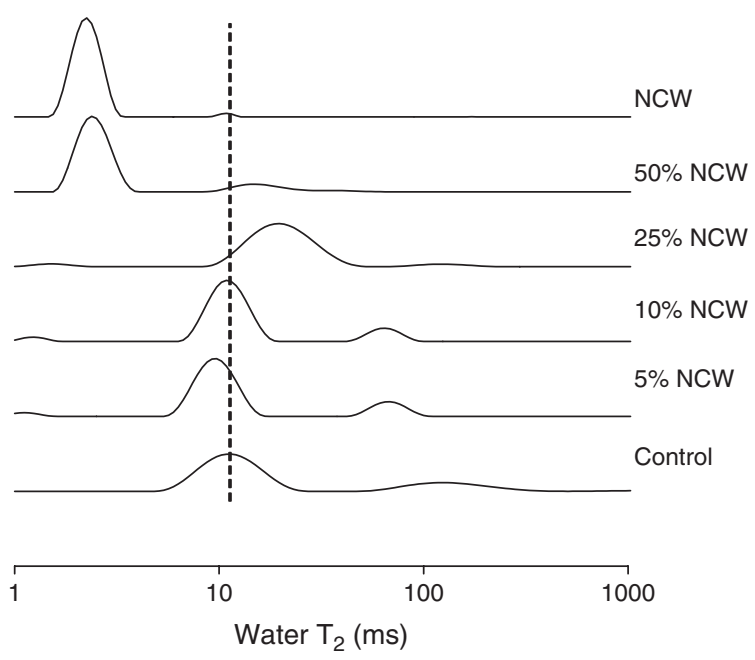

Fig. 3. Distribution of spin-spin relaxation times of absorbed water within control xylan/sorbitol, pure NCW and nanocomposite films reinforced with $10 \%, 17 \%, 25 \%, 35 \%$ and $50 \%$ NCW produced via ILTs of ${ }^{1} \mathrm{H}$ CMPG NMR $\mathrm{T}_{2}$ experiments conditioned under $100 \% \mathrm{RH}$ for $48 \mathrm{~h}$. The vertical dotted lines serve to visually demonstrate shifts in peak position.

J. Nanosci. Nanotechnol. 11, 1-8, 2011 scattering from the amorphous parts. The XRD pattern of the mechanically mixed particles of xylan and sorbitol shows a very comparable pattern with sorbitol particles (see Figs. 4 and 5). The observed intensity for this mixture drops significantly compared to sorbitol pattern, because of the semi-crystalline nature of xylan particles. After solution casting the control xylan/sorbitol film, the crystallinity decreases significantly with respect to the xylan and sorbitol particle mixture.

In Figure 5, the effects of making the $25 \%$ xylan/ $\mathrm{NCW}$ nanocomposite film, on crystallinity of the initial components, have been shown. XRD patterns of xylan/sorbitol particles show the strong sorbitol crystallinity. In Figure 5, $25 \% \mathrm{NCW}$ has been added to the mixture of xylan-sorbitol and the peaks near $22^{\circ}$ have been enhanced due to the NCW crystalline peak at $22.2^{\circ}$. The nanocomposite film with $25 \% \mathrm{CNW}$ shows a much lower crystallinity than the sample of mechanically mixed components, but its dominant peaks come from NCW peaks. The XRD of mechanically mixed xylan, sorbitol, NCWs shows a superposition of the diffraction patterns from crystalline sorbitol, NCW and semi-crystalline xylan. This result indicates that dry mixing does not affect the individual film components on the atomic scale whereas the film formation procedure of the xylan film with NCW does.

The morphology of xylan-NCW nanocomposite films was then further studied using XRD focusing on specific

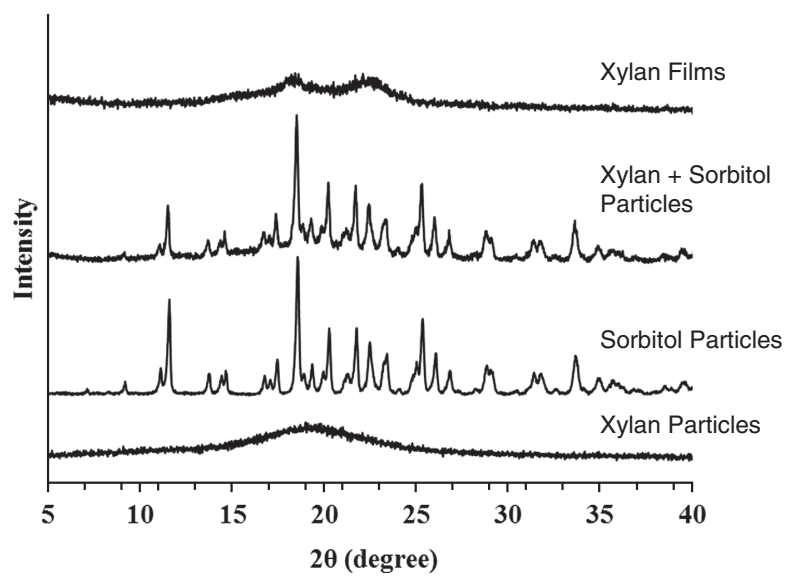

Fig. 4. Wide-angle X-ray diffraction patterns of xylan particles, sorbitol particles, mechanical mix of 1:1 mass ratio of xylan and sorbitol particles, and control xylan/sorbitol film. 


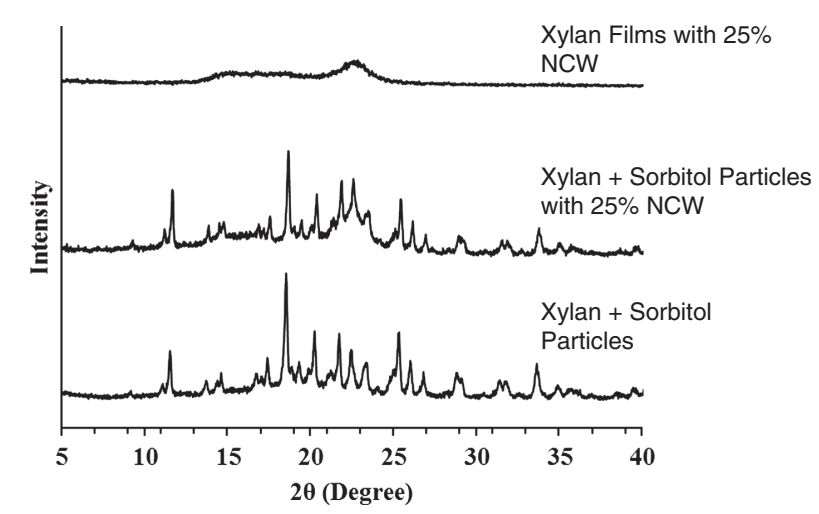

Fig. 5. Wide angle X-ray diffraction patterns of mechanically mixed 1:1 xylan and sorbitol, mechanically mixed 1:1 xylan and sorbitol with $25 \% \mathrm{NCW}$, and xylan/sorbitol film with $25 \% \mathrm{NCW}$ generated by stirring, heating and solution casting.

crystalline peaks associated with cellulose and xylan. The diffractograms of xylan-NCW nanocomposite films with different whisker contents are shown in Figure 6. The control xylan/sorbitol film shows weak crystalline peaks at $2 \theta=17.9$ and $22.2^{\circ}$ which is in agreement with literature. ${ }^{21,46}$ Similar to the control xylan/sorbitol film, the nanocomposite of $10 \% \mathrm{NCW}$ shows weak and broad diffraction pattern. As the NCW concentration increases, the patterns show characteristic diffractions of cellulose I at $2 \theta=16.1$ and $22.6^{\circ}$ that correspond to (110) and (200) planes. $^{44,21}$ The diffraction peak at $22.2^{\circ}$ has contribution from both xylan in the film matrix and NCWs, so it becomes noticeably sharper as the loading increases. However, a second peak attributed to xylan in the control xylan/sorbitol film occurs at a slightly higher angle $\left(\sim 17.9^{\circ}\right)$ than the diffraction peak associated with the 110 planes in NCWs. This second peak appears as a very broad signal in the nanocomposite films and seems to increase with NCW loading, suggesting an increase in crystallinity of the xylan in the matrix film may be related to increased NCW incorporation.

An overall increase in the relative amount of crystalline material in the nanocomposite films, a indicated by the change in the diffraction peak at $22.2^{\circ}$, with

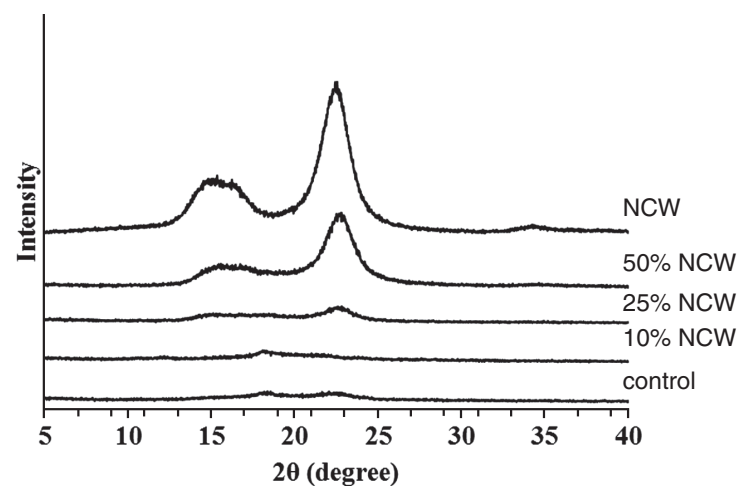

Fig. 6. Wide-angle X-ray diffraction patterns of NCW/xylan nanocomposite films of increasing whisker content. increased whisker incorporation was expected and consistent with work by other researchers. ${ }^{44-46}$ Accordingly, we believe that the higher relative amount of crystalline material in the nanocomposites results in less penetration of water molecules into the film. This would then result in lower water transmission rate and may in part explain the observed improved barrier properties of the nanocomposite film. The angle of the xylan crystalline peak at $\sim 17.9^{\circ}$ in the diffractograms of the nanocomposite films also seems to change to lower values when compared to the control xylan/sorbitol film. The distance between the lattice planes, $d$, can be related to the Bragg angle, $\theta$, using Bragg's law: $2 d \sin \theta=n \lambda$, where $n$ is an integer and $\lambda$ is the wavelength of the $\mathrm{X}$-ray radiation. A lower angle correlates to a larger distance between the lattice planes. This may be to due to an altering of the crystal structure of xylan in the nanocomposite during film formation and/or more likely, changed contributions of overlapping diffraction peaks for NCWs and xylan at 16.1 and $17.9^{\circ}$, respectively.

Crystallinity Index (CI) has been calculated for the xylan/sorbitol films with different NCW loading using the XRD amorphous subtraction method. ${ }^{47}$ The basis for this method was outlined by Ruland et al. ${ }^{48}$ who determined crystallinity by subtracting the amorphous contribution from diffraction spectra using an amorphous standard. ${ }^{48}$ The challenge for this method is to select an amorphous standard that is similar to the amorphous component in the sample. Various materials have been used as an amorphous standard, such as ball-milled cellulose, and xylan powder. Xylan powder was used as the standard in our measurements. A scale factor is applied to the spectrum of the amorphous material, so that after subtraction of the amorphous spectrum from the original spectrum, no part of the residual spectrum contains a negative signal. Figure 7 shows the XRD spectrum of xylan films containing 0 (control xylan/sorbitol), 10, 25, 50\% NCW and pure NCW $(100 \%)$ along with their scaled amorphous spectrum. The difference between the XRD spectrum and the amorphous spectrum denotes the crystalline contribution part of the spectrum. CI is calculated as the ratio between the area of the crystalline contribution and the total area of the spectrum from $5-40^{\circ}$.

Table II shows the calculated crystallinity index for xylan films containing 0 (control xylan/sorbitol), 10, 25, $50 \% \mathrm{NCW}$ and pure NCW (100\%). The CI values are 26, $32,42,61$, and $69 \%$ respectively. Control xylan/sorbitol film with no NCW loading shows some crystallinity (26\%) most likely a consequence of the film formation procedure. Table II also shows that a pure NCW film has a CI $67 \%$, very comparable to that determined by NMR, attributed to unhydrolyzed amorphous cellulose and cellulose at crystallite surfaces. Assuming film formation causes no change to the xylan/sorbitol matrix, one may expect to calculate the $\mathrm{CI}$ of the composite based on a simple additive rule 


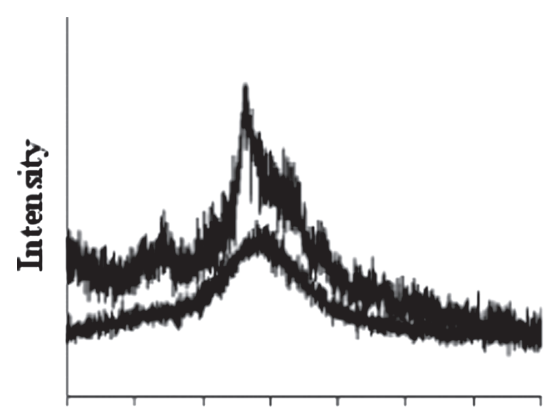

$\begin{array}{llllllll}5 & 10 & 15 & 20 & 25 & 30 & 35 & 40\end{array}$

$2 \theta$ (degree)

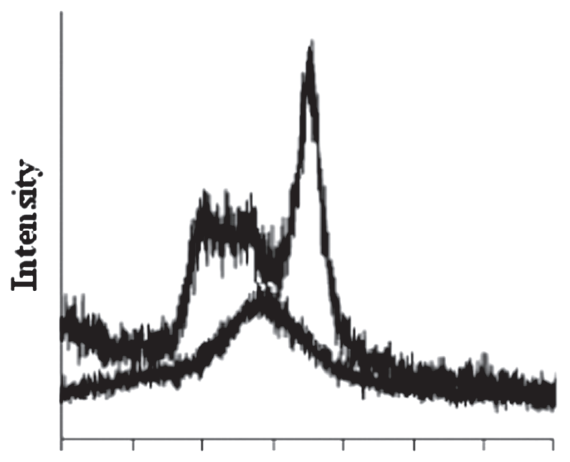

$\begin{array}{llllllll}5 & 10 & 15 & 20 & 25 & 30 & 35 & 40\end{array}$

$2 \theta$ (degree)
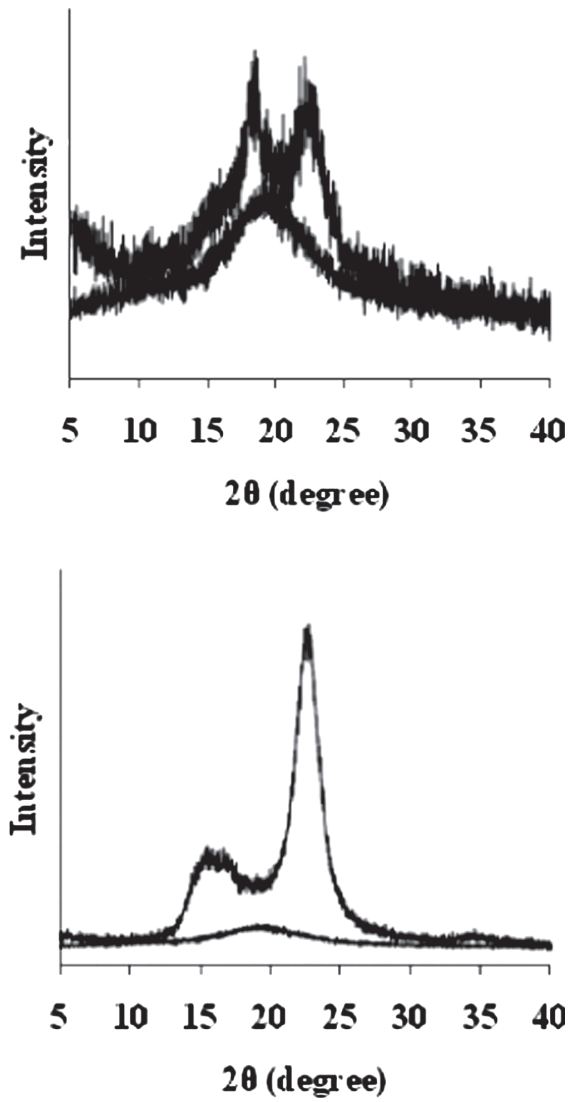

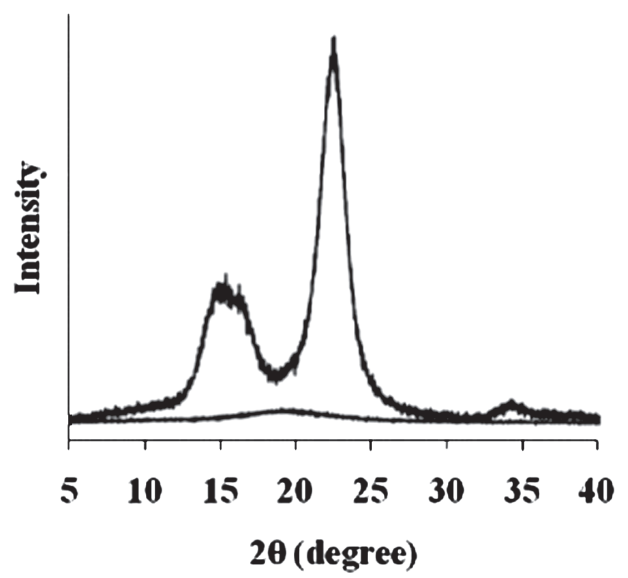

Fig. 7. XRD spectrum of NCW and xylan films containing 0 (control xylan/sorbitol), 10, 25, 50\% NCW and pure NCW (100\%) along with their scaled amorphous spectrum.

Table II. Crystallinity index (CI) for xylan/sorbitol nanocomposites with different NCW contents.

\begin{tabular}{lccc}
\hline Sample & $\begin{array}{c}\text { CI by XRD amorphous } \\
\text { subtraction method (\%) }\end{array}$ & $\begin{array}{c}\text { Expected CI by } \\
\text { mixing rule (\%) }\end{array}$ & $\begin{array}{c}\text { Difference } \\
(\%)\end{array}$ \\
\hline Control film & 26 & - & - \\
$10 \% \mathrm{NCW}$ & 32 & 31 & +1 \\
$25 \% \mathrm{NCW}$ & 42 & 37 & +5 \\
$50 \% \mathrm{NCW}$ & 61 & 48 & +13 \\
NCW & 69 & - & - \\
\hline
\end{tabular}

J. Nanosci. Nanotechnol. 11, 1-8, 2011 of mixtures, the NCW volume fraction and the CI of pure NCW and control xylan/sorbitol film.

In Table II, as expected, the crystallinity index of the films increases as the NCW loading increases in the films. The results seem to suggest that there is more crystalline material in the nanocomposite than can be directly correlated with the addition of NCWs and that the nanocomposite deviates from the rule of mixtures. This is significant because these results suggest that there are significant interactions between the xylan film and NCW that increases the crystallinity specifically in the matrix of the film. 


\section{CONCLUSIONS}

A processing-structure-property relationship study of xylan-NCWs nanocomposites was done and the effectiveness of nanocellulose as reinforcement was demonstrated. Degradable polymer nanocomposites from renewable resources based on xylan and nano cellulose whiskers were synthesized by solution processing techniques. The spectral data obtained for the NCW/xylan nanocomposite films showed that the amount of xylan adsorbed to cellulose increases with the addition of NCW in the matrix. In addition, NMR $T_{2}$ relaxation experiments studies were conducted to investigate the change in the nature of carbohydrate-water interactions as a result of NCW incorporation. These results will facilitate an improved understanding of the mechanisms involved in the superior barrier and mechanical properties of rigid hydrogen bonded xylanwhisker nanocomposite films previously reported. XRD studies show that when a xylan-whisker nanocomposite films is formed the mixing occurs on the atomic scale and NCW loading increases the matrix crystallinity.

Acknowledgments: The authors wish to acknowledge the support of the member companies of IPST at the Georgia Institute of Technology and the IPST Fellowship. Portions of this work are being used by Amit Saxena for his $\mathrm{PhD}$ requirements for graduation from the School of Chemistry and Biochemistry at the Georgia Institute of Technology, Atlanta, Georgia.

\section{References and Notes}

1. L. Shen, E. Worrell, and M. Patel, Biofuels, Bioprod. Biorefin. 4, 25 (2010).

2. A. Samir, F. Alloin, and A. Dufresne, Biomacromolecules 6, 612 (2005).

3. A. J. Ragauskas, C. K. Williams, B. H. Davison, G. Britovsek, J. Cairney, C. A. Eckert, W. J. Frederick, J. P. Hallett, D. J. Leak, C. L. Liotta, J. R. Mielenz, R. Murphy, R. Templer, and T. Tschaplinski, Science 311, 484 (2006).

4. L. Petersson and K. Oksman, Compos. Sci. Technol. 66, 2187 (2006).

5. L. Shen, E. Worrell, and M. Patel, Biofuels, Bioprod. Biorefin. 4, 25 (2010).

6. A. Samir, F. Alloin, and A. Dufresne, Biomacromolecules 6, 612 (2005).

7. A. J. Ragauskas, C. K. Williams, B. H. Davison, G. Britovsek, J. Cairney, C. A. Eckert, W. J. Frederick, J. P. Hallett, D. J. Leak, C. L. Liotta, J. R. Mielenz, R. Murphy, R. Templer, and T. Tschaplinski, Science 311, 484 (2006).

8. L. Petersson and K. Oksman, Compos. Sci. Technol. 66, 2187 (2006).

9. A. Gennadios, M. A. Hanna, and B. Kurth, LWT-Food Sci. Technol. 30, 337 (1997).

10. K. N. Turhan and F. J. Sahbaz, Food Eng. 61, 459 (2004)

11. N. Gontard, S. Guilbert, and J. L. Cuq, J. Food Sci. 57, 190 (1992).

12. G. S. Banker, J. Pharm. Sci. 55, 81 (1966)

13. M. P. Coughlan and G. P. Hazlewood, Hemicellulose and Hemicellulases (Eds). Portland Press Ltd., NC, U.S.A (1993).

14. A. Ebringerová and T. Heinze, Macromol. Rapid Commun., 21, 542 (2000).
15. I. Gabrielli and P. Gatenholm, J. Appl. Polym. Sci, 69, 1661 (1998).

16. J. Hartman, A. C. Albertsson, M. S. Lindblad, and J. Sjöberg, J. Appl. Polym. Sci. 100, 2985 (2006).

17. M. Gröndahl, L. Eriksson, and P. Gatenholm, Biomacromolecules 5, 1528 (2004)

18. P. Dole, C. Joly, E. Espuche, I. Alric, and N. Gontard, Carbohydr. Polym. 58, 335 (2004).

19. B. L. Butler, P. J. Vergano, R. F. Testin, J. M. Bunn, and J. L. Wiles, J. Food Sci. 61, 953 (1996).

20. I. Arvanitoyannis and C. G. Biliaderis, Carbohydr. Polym. 38, 47 (1999)

21. M. Gröndahl, L. Eriksson, and P. Gatenholm, Biomacromolecules 5, 1528 (2004)

22. A. Linder, R. Bergman, A. Bodin, and P. Gatenholm, Langmuir, 19, 5072 (2003)

23. S. P. Miranda, O. Garnica, V. Lara-Sagahon, and G. Cárdenas, J. Chil. Chem. Soc. 49, 173 (2004).

24. M. A. S. A. Samir, F. Alloin, and A. Dufresne, Biomacromolecules 6, 612 (2005).

25. I. Kvien, J. Sugiyama, M. Votrubec, and K. Oksman, J. Mater. Sci. 42, 8163 (2007).

26. L. Petersson, I. Kvien, and K. Oksman, Composites Sci. Technol. 67, 2535 (2007).

27. X. D. Cao, H. Dong, and C. M. Li, Biomacromolecules 8, 899 (2007).

28. S. Harbaugh, N. K. Loughnane, M. Davidson, L. Narayanan, S. Trott, Y. G Chushak, and M. O. Stone, Biomacromolecules 6, 1055 (2005).

29. C. O. Oriakhi, Chem. Br. 34, 59 (1998)

30. Y. Imai, S. Nishimura, E. Abe, H. Tateyama, A. Abiko, A. Yamaguchi, T. Aoyama, and H. Taguchi, Chem. Mater. 14, 477 (2002).

31. A. Saxena, T. Elder, P. Shaobo, and A. J. Ragauskas, Composite Part B 40, 727 (2009).

32. A. Saxena and A. J. Ragauskas, Carbohydr. Polym. 78, 357 (2009).

33. A. Saxena, T. Elder, J. Kenvin, and A. J. Ragauskas, Nano-Micro Letters 2, 235 (2010)

34. P. Larsson, E. Hult, K. Wickholm, E. Pettersson, and T. Iversen, Solid State Nucl. Magn. Reson. 15, 31 (1999).

35. T. Liiti, S. L. Maunu, B. Hortling, T. Tamminen, O. Pekkala, and A. Varhim, Cellulose 10, 307 (2003)

36. D. Bhattacharya, L. T. Germinario, and W. T. Winter, Carbohydr. Polym. 73, 371 (2008).

37. P. Larsson, K. Wickholm, and T. Iversen, Carbohydr. Res. 302, 19 (1997)

38. R. H. Newman and J. A. Hemmingson, Cellulose 2, 95 (1995).

39. M. Lopez, H. Bizot, G. Chambat, M. Marais, A. Zykwinska, M. Ralet, H. Driguez, and A. Buleon, Biomacromolecules 11, 1417 (2010)

40. C. G. Mothe and M. B. Tavares, Polym. Degrad. Stabil. 61, 253 (1998).

41. R. Menon, A. L. MacKay, A. E. Burgess, and J. S. Swanson, J. Appl. Polym. Sci. 33, 1141 (1987).

42. C. D. Araujo, A. L. MacKay, K. P. Whittall, and J. R. Hailey, J. Magn. Reson., Ser. B 101, 248 (1993).

43. M. Foston and A. J. Ragauskas, Energy Fuels 24, 5677 (2010).

44. Q. Li, J. Zhou, and L. Zhang, J. Polym. Sci., Part B: Polym. Phys. 47, 1069 (2009).

45. J. M. Lagaron, R. Catala, and R. Gavaa Mater. Sci. Technol. 20, 1 (2004).

46. K. S. Mikkonen, A. P. Mathew, K. Pirkkalainen, R. Serimaa, C. Xu, S. Willfor, K. Oksman, and M. Tenkanen, Cellulose 17, 69 (2010).

47. S. Park, J. O Baker, M. E Himmel, P. A. Parilla, and D. K. Johnson, Biotechnol. Biofuels 3, 10 (2010).

48. W. Ruland, Acta Cryst. 14, 1180 (1961).

Received: 15 June 2011. Accepted: 5 August 2011. 